\title{
Role of CGRP pathway polymorphisms in migraine: a systematic review and impact on CGRP mAbs migraine therapy
}

\author{
Damiana Scuteri ${ }^{1,2}$, Maria Tiziana Corasaniti ${ }^{3}$, Paolo Tonin² ${ }^{2}$ Pierluigi Nicotera ${ }^{4}$ and Giacinto Bagetta ${ }^{\text {* }}$
}

\begin{abstract}
Background: the interest of clinical reaseach in polymorphisms and epigenetics in migraine has been growing over the years. Due to the new era of preventative migraine treatment opened by monoclonal antibodies (mAbs) targeting the signaling of the calcitonin-gene related peptide (CGRP), the present systematic review aims at identifying genetic variants occurring along the CGRP pathway and at verifying whether these can affect the clinical features and the course of disease and the responsiveness of patients to therapy.
\end{abstract}

Methods: the literature search has been conducted consulting the most relevant scientific databases, i.e. PubMed/ MEDLINE, Scopus, Web of Science, the Human Genome Epidemiology (HuGE) Published Literature database (Public Health Genomics Knowledge Base) and Clinicaltrials.gov from database inception until April 1, 2021. The process of identification and selection of the studies included in the analysis has followed the PRISMA (Preferred Reporting Items for Systematic reviews and Meta-Analyses) criteria for systematic reviews and meta-analyses and the guidance from the Human Genome Epidemiology Network for reporting gene-disease associations.

Results: the search has retrieved 800 results, among which only 7 studies have met the eligibility criteria for inclusion in the analysis. The latter are case-control studies of genetic association and an exploratory analysis and two polymorphisms have been detected as the most recurring: the rs3781719 ( $T>C)$ of the CALC A gene encoding CGRP and the rs7590387 of the gene encoding the receptor activity-modifying protein (RAMP) 1 (C > G). Only one study assessing the methylation pattern with regard to CGRP pathway has been found from the search. No genetic association studies investigating the possible effect of genetic variants affecting CGRP signaling on the responsiveness to the most recent pharmacological approaches, i.e. anti-CGRP(R) mAbs, gepants and ditans, have been published. According to the Human Genome Epidemiology (HuGE) systematic reviews and meta-analyses riskof-bias score for genetic association studies, the heterogeneity between and across studies and the small sample size do not allow to draw conclusions and prompt future studies.

Conclusions: adequately powered, good quality genetic association studies are needed to understand the impact of genetic variants affecting the pathway of CGRP on migraine susceptibility and clinical manifestation and to predict the response to therapy in terms of efficacy and safety.

Keywords: polymorphisms, SNPs, methylation, epigenetic, migraine, CGRP, CALC A, RAMP 1, CLR, RCP, CALCRL, AMYLIN-1, systematic review

\footnotetext{
*Correspondence: g.bagetta@unical.it

'Pharmacotechnology Documentation and Transfer Unit, Preclinical and

Translational Pharmacology, Department of Pharmacy, Health and Nutritional

Sciences, University of Calabria, 87036 Rende, Italy

Full list of author information is available at the end of the article
}

\section{$\triangle B M C$}

C C The Author(s). 2021 Open Access This article is licensed under a Creative Commons Attribution 4.0 International License, which permits use, sharing, adaptation, distribution and reproduction in any medium or format, as long as you give appropriate credit to the original author(s) and the source, provide a link to the Creative Commons licence, and indicate if changes were made. The images or other third party material in this article are included in the article's Creative Commons licence, unless indicated otherwise in a credit line to the material. If material is not included in the article's Creative Commons licence and your intended use is not permitted by statutory regulation or exceeds the permitted use, you will need to obtain permission directly from the copyright holder. To view a copy of this licence, visit http://creativecommons.org/licenses/by/4.0/ The Creative Commons Public Domain Dedication waiver (http://creativecommons.org/publicdomain/zero/1.0/) applies to the data made available in this article, unless otherwise stated in a credit line to the data. 


\section{Background}

\section{Rationale and objective}

Migraine is a primary headache disorder defined as a prevalent neurologic disease characterized by headaches that can occur with or without aura, consisting of transient focal neurological symptoms (visual, sensory, speech and/or language, motor, brainstem and retinal) that usually precede by hours or days, or sometimes accompany, the headache [1]. The social worldwide burden of migraine is noteworthy since it ranks the sixth most prevalent disease and the second cause of disability worldwide [2], accounting for around $7 \%$ of all-cause Years Lived with Disability (YLD) and for $72 \%$ of all YLDs associated to neurological disorders [3]. In fact, according to report from the Global Burden of Disease Headache in 2018, $14.4 \%$ of the global population suffers from migraine making of it the global second leading cause of disability [4]. Migraine belongs to the category of chronic diseases since it is characterized by episodic manifestations (CDEM) [5] that can undergo chronification in the process of clinical transformation and progression [6]. The prevention of the episodic attacks is fundamental to avoid chronification. The nociceptors from the dura mater and periorbital skin project [7] to second-order neurons in the trigeminal nucleus caudalis $[8,9]$, that can be subjected to sensitization as third-order neurons mainly in the pulvinar of the thalamus. The sensitization of the latter induces cutaneous allodynia, cephalic at the beginning and generalized or extracephalic at later stage $[8,9]$ and it is involved in chronification. The vasodilatory neuropeptides are remarkably implicated in the latter dural neurogenic inflammation. Among these, calcitoningene related peptide (CGRP) is the the most important player responsible for clinically relevant vasodilation acting on its receptor in the trigeminal ganglion [10] (Fig. 1).

In particular, the $\alpha$-CGRP encoded by the CALC A (or CALC I) gene is involved in the pathogenesis of migraine [12]. The effects of CGRP are mediated by its interaction with the CGRP receptor, a $G \alpha_{\text {s }}$ proteincoupled receptor formed by the calcitonin receptorlike receptor (CLR), the receptor activity-modifying protein (RAMP) 1 and the receptor component protein (RCP) [13].CGRP requires also the fusion protein of the extracellular domains of human $G$ protein-coupled receptor calcitonin receptor-like receptor CALCRL to activate the downstream signaling that ends with vasodilation [14]. Apart from this canonical receptor, CGRP signal transduction is mediated by the second receptor that is the human amylin subtype 1 receptor (AMY1). The genes encoding the latter molecules responsible for CGRP-induced signaling are subjected to genetic variants influencing their activities. Specific anti-migraine drugs for acute treatment of attacks, e.g. triptans, are agonists of 5-HT1B, 5-HT1D and 5-HT1F that finally inhibit CGRP release during migraine attacks [12]. Furthermore, novel therapeutic and preventative approaches target the CGRP signaling: these are the gepants, antagonists of CGRP receptor, and the anti-CGRP(R) monoclonal antibodies (mAbs) [15]. Although the anti-CGRP(R) mAbs are the first specific preventive therapy which can provide pain relief to difficult-to-treat patients [12], some $40 \%$ of the latter are non responders [16]. Apart from the monogenic forms of migraine and the evidence of rare pathologic genetic variants, several single-nucleotide polymorphisms (SNPs) have been associated with differences in migraine susceptibility, clinical features and response to treatment. For instance, genes related to vascular modifications and cardiovascular diseases, e.g.

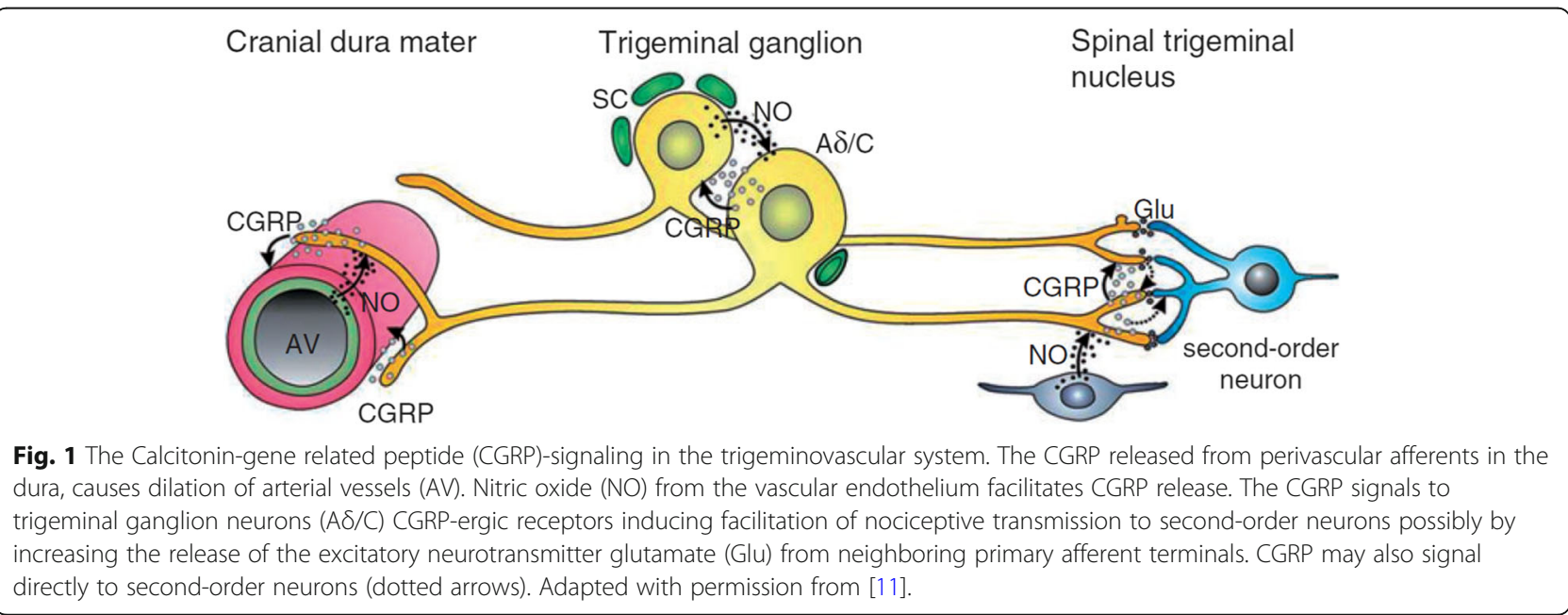


SNPs of the gene OMIM encoding the angiotensin converting enzyme (ACE) or SNPs of the methylenetetrahydrofolate reductase (MTHFR) genetic variants have been implicated in the susceptibility to migraine and aura and in the frequency of migraine attacks. The human leukocyte antigens (HLA) have been associated in heredity for migraine [17] and the HLA Class II DR2 antigen has been found to have a protective role toward migraine with aura $[18,19]$. Interestingly, some SNPs have been associated with responsiveness to drugs and, thus, to tendency to chronification with overuse and medications overuse headache $(\mathrm{MOH})$ [20]. Thus, in the future genetic profiling will be fundamental to foresee the efficacy and safety of therapy, depending on individual genetic variability $[21,22]$ and to design new drugs tailored on each patient's genetics [23]. For example, the SNP C825TC of rs5443 in the gene GNB3 coding the G protein $\beta 3$ subunit in the signaling of $5 \mathrm{HT}_{1 \mathrm{~B} / 1 \mathrm{D}}$ is a common genetic variant implicated in the rate of good responsiveness to triptans $[24,25]$. Other SNPs affecting the destiny of triptans are crucial to their pharmacokinetics and pharmacodynamics. The enzymes responsible for triptan degradation monoamine oxidase (MAO) A and the cytochrome CYP1A2 influence the response to triptans [25] and the SNP rs4680 of the catechol-Omethyltransferase (COMT) increases the risk of poor response to frovatriptan [26]. Therefore, SNPs occurring along the CGRP receptor pathway could affect the clinical evolution of migraine and, thus, might influence to some extent the responsiveness to antiCGRP(R) mAbs [20]. In particular, for mAbs directed towards CGRP and its receptor the affinity for the functional receptor effectors Fc receptors is fundamental for the maintenance of the antibody-ligand complex and for the mAb elimination half-life [27]. Fc $\gamma$ Rs are responsible for antibody-dependent cell-mediated cytotoxicity (ADCC) in cancer therapy [28, 29], e.g. rituximab. In fact, they can influence the affinity between the Fc $\gamma$ Rs and the IgG [30] and the alteration of this mechanism can be of fundamental importance for the effectiveness of anti-CGRP/CGRP(R) mAbs. Polymorphisms and gene copy-number variations (CNVs) of FcRs have been associated to the efficacy of mAbs, as it may occur for trastuzumab [31]. Finally, epigenetic modifications, including DNA methylation and post-translational modifications of the histones tails, have been implicated in modulation of attack frequency [32]. The aim of this systematic review is to assess whether there are SNPs or methylation patterns along the CGRP pathway that can influence susceptibility to migraine, with and without aura, frequency and severity of attacks and responsiveness to treatment.

\section{Methods}

\section{Objectives and protocol}

To our knowledge the present systematic review is the first designed to verify the working hypothesis that the SNPs or methylation patterns occurring along the CGRP pathway can affect the clinical features and the course of disease and the responsiveness of patients affected to anti-migraine therapy. In order to address this PICOS (participants/population, interventions, comparisons, outcomes, and study design) question, the PRISMA recommendations $[33,34]$ and the guidance from the Human Genome Epidemiology Network for reporting gene-disease associations [35] have been followed. The possibility of SNPs to account for some lack of response to anti-CGRP(R) mAbs, mainly, and to anti-migraine therapy, in general, is a broad question still representing a lack of knowledge; therefore, this work will provide an overview of evidence, including all the existing studies investigating direct genetic association, for assessing the consistency of the body of evidence to prompt future research. For this reason, the protocol has not been registered in the International prospective register of systematic reviews PROSPERO. The systematic review and meta-analysis has been conducted in accordance to a protocol established prior to the literature search. The retrieved results have been evaluated and doublechecked independently by two researchers. Any conflicts have been resolved by a third author.

\section{Inclusion criteria}

The analysis included genetic association studies assessing the direct genetic association of SNPs or epigenetic modifications affecting genes involved in the CGRP pathway on the following aspects: susceptibility to migraine, with and without aura; frequency and severity of attacks; responsiveness to treatment. No filters about study duration or follow-up and no restrictions concerned with publication date have been applied. In vitro and in vivo animal studies, narrative or systematic reviews and meta-analysis, abstracts and congress communications, proceedings, editorials and book chapters as well as studies not available in full text and not published in English have been excluded from the analysis.

\section{Information sources}

The literature search has been performed consulting the most relevant scientific databases, i.e. PubMed/MEDLINE, Scopus, Web of Science, the Human Genome Epidemiology (HuGE) Published Literature database (Public Health Genomics Knowledge Base) and Clinicaltrials.gov. The search could not be conducted also on Embase since it was not freely/institutionally available. No restriction of publication date has been applied. The 
databases have been searched for records matching the search strings used from their inception to May, 212021 that was the date of last search.

\section{Search strategy}

The following Medical Subject Headings (MeSH) terms and modifications have been used as search terms in combination: "migraine", "CGRP", "calcitonin generelated peptide", "CGRP receptor", "CALC A", "CALC I", "RAMP 1", "CALCRL", "CLR", "RCP”, "AMYLIN-1", "AMYLIN-1 receptor", "polymorphisms", "SNP(s)", "epigenetic", "methylation", "anti-CGRP(R) monoclonal antibodies", "anti-CGRP(R) mAbs", "triptans", "gepants", "ditans".

\section{Studies selection}

The assessment of the inclusion and exclusion criteria and the determination of eligibility of the studies has been carried out independently by two authors for minimizing the risk to exclude relevant records. Duplicate records have been eliminated and the following first screening has assessed the title and abstract. Then, the full text has been evaluated for inclusion in qualitative and/or in quantitative synthesis. The references list of the articles has been evaluated in order to extend and refine the search. Complete consensus among all the authors has been achieved without relevant conflicts planned to be solved through the Delphi method [36].

\section{Data analysis}

The synthesis of the results has been conducted according to the Cochrane Consumers and Communication Review Group guidelines [37]. The assessment of the risk of bias and of the quality of retrieved studies has been performed in agreement to Human Genome Epidemiology (HuGE) systematic reviews and meta-analyses risk-of-bias score for genetic association studies [38]. Hence, the latter score ranges from low to unclear and high risk, taking into account the following 4 outcomes rated yes, no or unclear: (1) Information bias - Accuracy of diagnosis of migraine and robustness of genotyping methods; (2) Confounding bias - Population stratification and other confounder effects; (3) Selective reporting of outcomes - reporting bias; (4) Hardy-Weinberg equilibrium (HWE) - assessment in the control groups. Sample size has been considered. Due to the heterogeneity of the studies and the difference of polymorphisms and outcomes investigated in the studies meeting the eligibility criteria, data concerned with odds ratios for genotypes could not be pooled and a genotype-based meta-analysis and assessment of the credibility of cumulative evidence through the Venice guidelines [39] have not resulted feasible.

\section{Results}

\section{Selection of the studies}

The search on the databases has retrieved 800 results: 396 records have been obtained form PubMed/MEDLINE, 209 from Scopus, 173 from Web of Science, 19 from the HuGE Published Literature database (Public Health Genomics Knowledge Base) and 3 from Clincaltrials.gov. The 800 records have been searched for duplicates. After duplicates removal there were 285 results to screen (also one of the three records obtained from Clincaltrials.gov has resulted to be a duplicate). The latter have been screened in title and abstract leaving 11 results to assess for eligibility. However, the study by An et al., 2017 [40] was not available in full text and the two records retrieved from Clincaltrials.gov are two studies without results since one is recruiting and the other not yet. In particular, the study INTERROGATE, Biomarker and Genetic Predictors of Erenumab Treatment Response (NCT04265755) is in the recruitment stage and it aims at exploring the relationship between clinical response to erenumab and genetic biomarkers, while the purpose of the BIOmarkers of MIGraine (BIOMIGA) proof of concept study (NCT04503083) is to detect biomarkers predictive of response to anti-CGRP(R) mAbs in severe migraineurs using, among others, pharmacogenetic evaluation and assessment of the methylation levels. Therefore, the full-text articles assessed for eligibility are 8 and 7 of them have met the inclusion criteria for qualitative analysis. In fact, the study by Louter et al., that considered the polymorphisms rs 2956 of CALC A gene and rs858745 of CALCRL gene possible candidate genes to be implicated in chronification [41] had to be excluded because of its different study design being a three stage genetic association study.

The process of identification and selection of the studies is illustrated in Fig. 2.

\section{Qualitative analysis}

The 7 articles eligible for analysis are grouped according to the gene of which the polymorphisms have been studied, based on the Cochrane Consumers and Communication Review Group guidelines. A summary of the main characteristics of the studies is reported in Table 1.

\section{Polymorphisms of the gene encoding CGRP}

The gene encoding CGRP has been studied for the first time in the study by Lemos et al., in a European population [42]. The SNP rs1553005 of the latter gene has been found to interact with the variant rs2049046 of the gene encoding brain-derived neurotrophic factor (BDNF) increasing the risk of migraine $[\mathrm{rs} 1553005(\mathrm{CGRP}) * \mathrm{rs} 2049046(\mathrm{BDNF})=\mathrm{GC} * \mathrm{AT}-\mathrm{OR}$ 1.88 (95\%CI 1.20-2.93), $P=0.005$ ] [42]. This is supported by the co-expression of the latter 


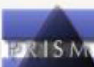

PRISMA 2009 Flow Diagram
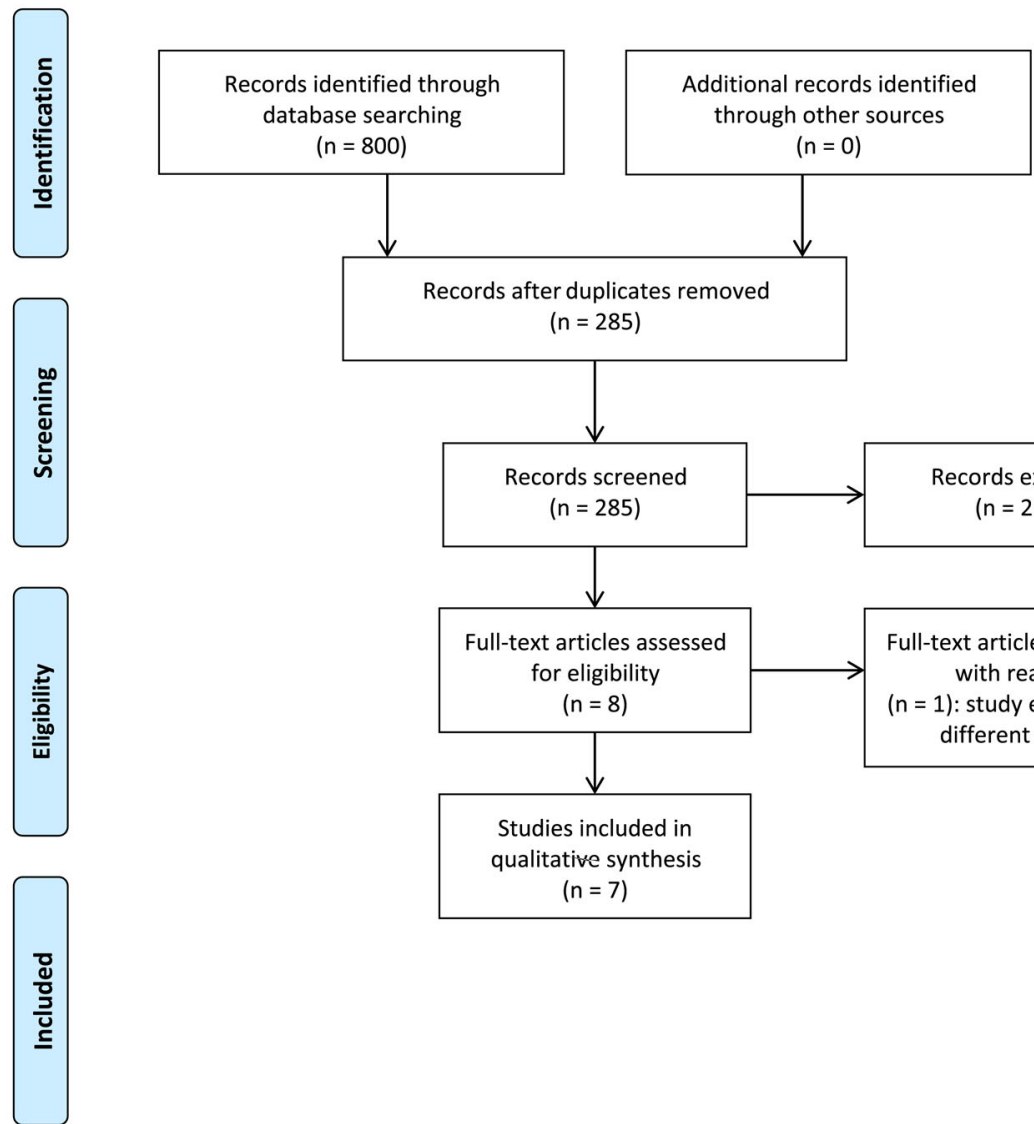

Records after duplicates removed

$(n=285)$

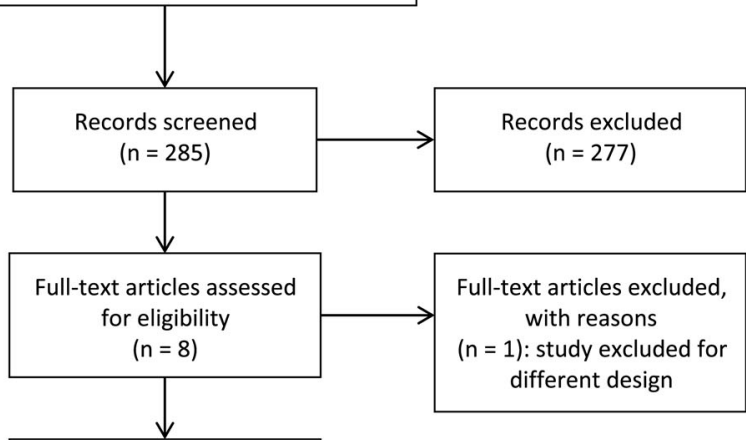

Studies included in

qualitative synthesis

$(n=7)$

Fig. 2 PRISMA flow diagram. PRISMA flow diagram reporting the process of identification and selection of the studies eligible for the systematic review and meta-analysis.

neurotransmitter that has been demonstrated in trigeminal ganglion neurons of rat. The study by Menon and collaborators [43] has investigated in an Australian population the role of the polymorphism rs35815751, consisting in a 16 bp deletion in the first intron of the CALC A gene that is a region with triplet G-run motifs, in the development of migraine with aura: no significant association between rs35815751 and migraine [for genotypes $(P=0.575)$ nor alleles $(P=0.502)]$, and migraine with aura (genotypes, $P=$ 0.666; alleles, $P=0.7$ ) or without aura (genotypes, $P=$ 0.325 ; alleles, $P=0.276$ ) has been found. The study of Guldiken and collaborators [44] has tested on female population the possible influence of the polymorphism rs3781719, consisting in T-692 C of CALC A gene, on attack frequency and severity and on the occurrence of aura, finding no significant association with migraine $(P=0.44)$ and aura $(P=0.52)$. Also in an Australian population, the study by Sutherland et al., [45] has investigated the possible correlation between the SNPs rs3781719 in the promoter region and rs145837941 in the coding sequence of CALC A and an increased susceptibility to migraine. None of the two polymorphisms have resulted associated to migraine susceptibility or with gender and the rs3781719 has not been associated to increased frequency of attacks or to the development of aura. This polymorphism has been studied also by Cargnin et al., in an Italian population for its influence on response to triptans in patients affected by migraine without aura and it has been tested for association with transformation into $\mathrm{MOH}$, providing no significant correlation [46]. However, CALC A rs3781719C allele has resulted to increase risk of lack of response to OnabotulinumtoxinA in a female population of Caucasian ethnicity and Spanish origin [OR (95 \%CI) 


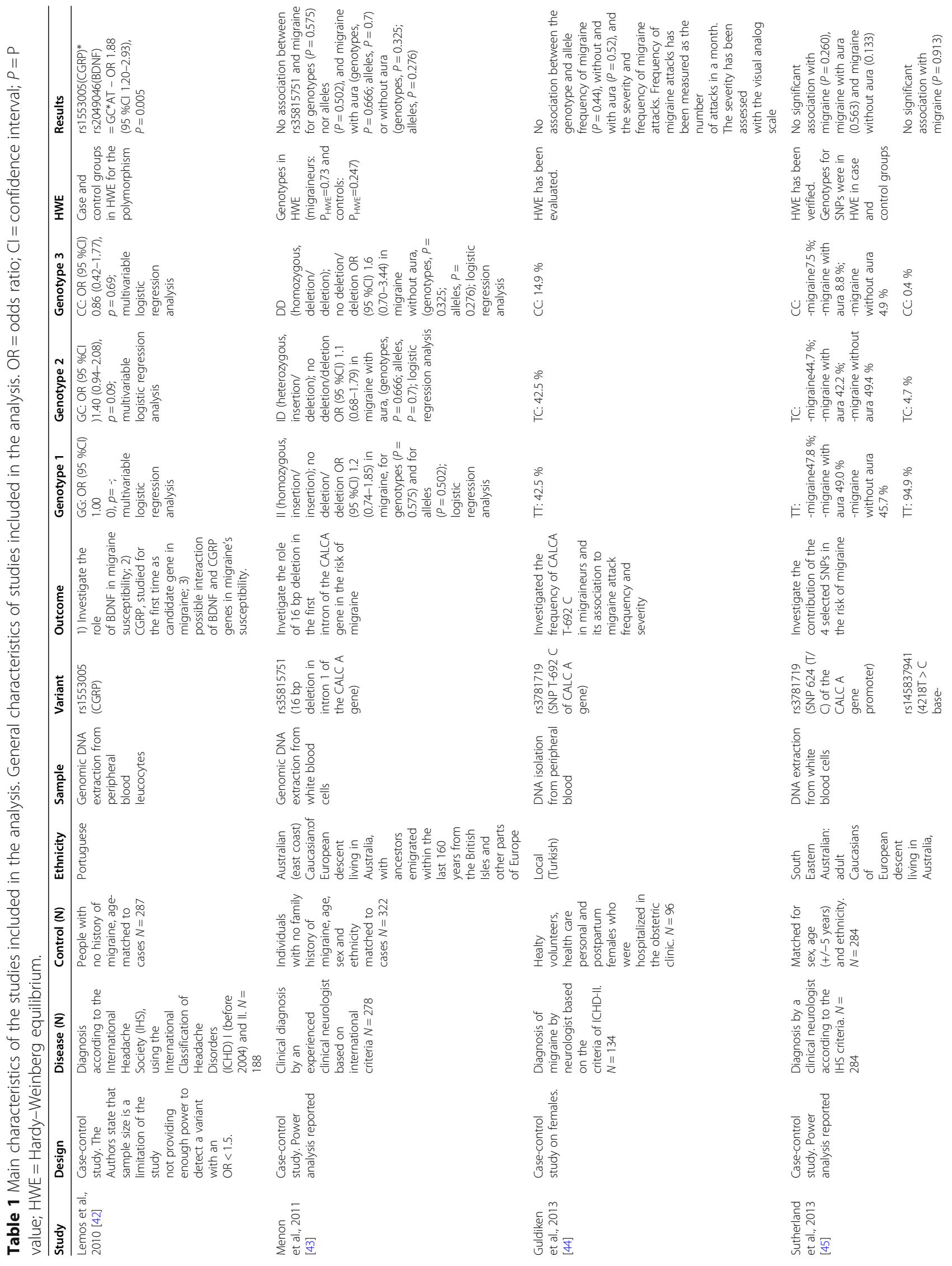




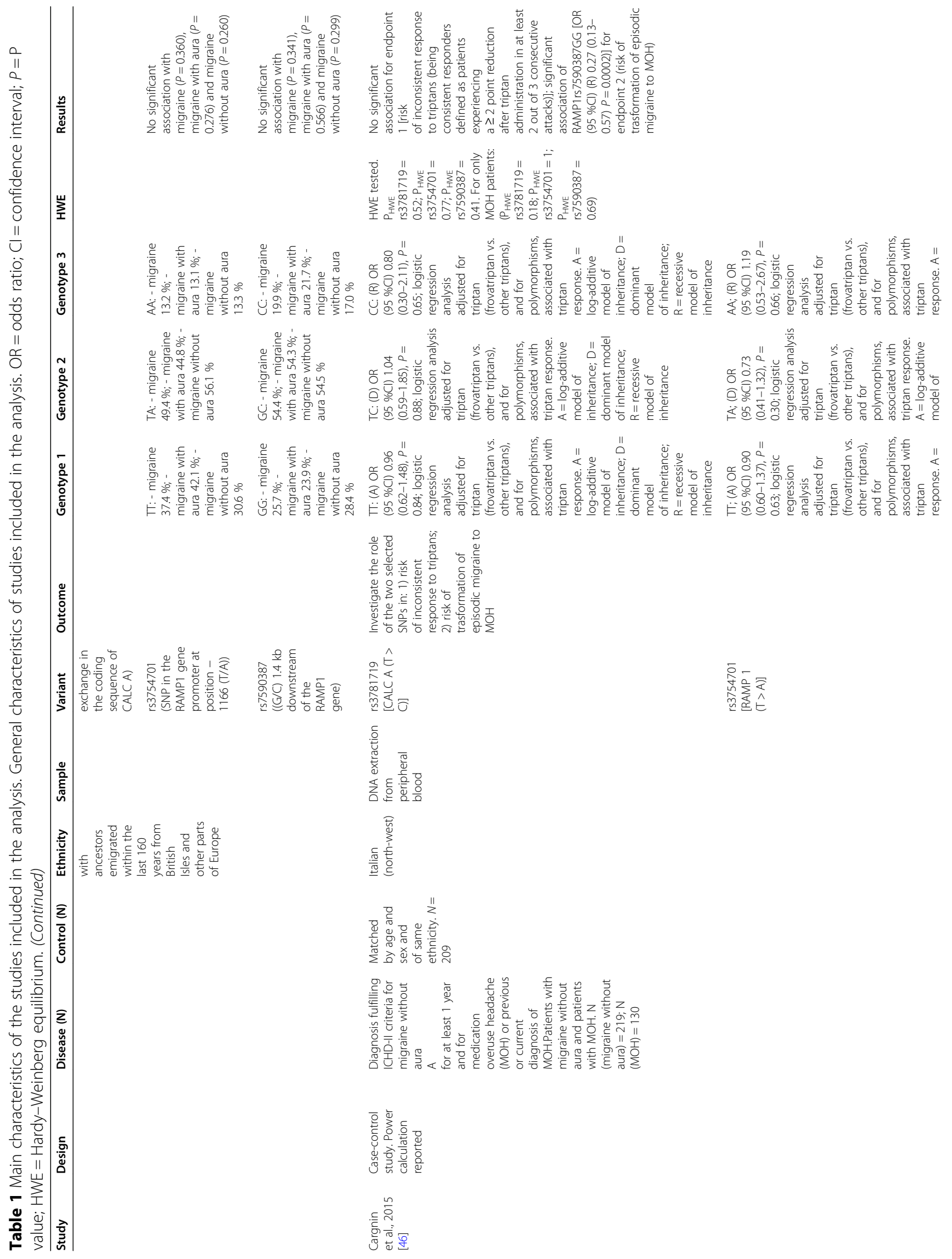




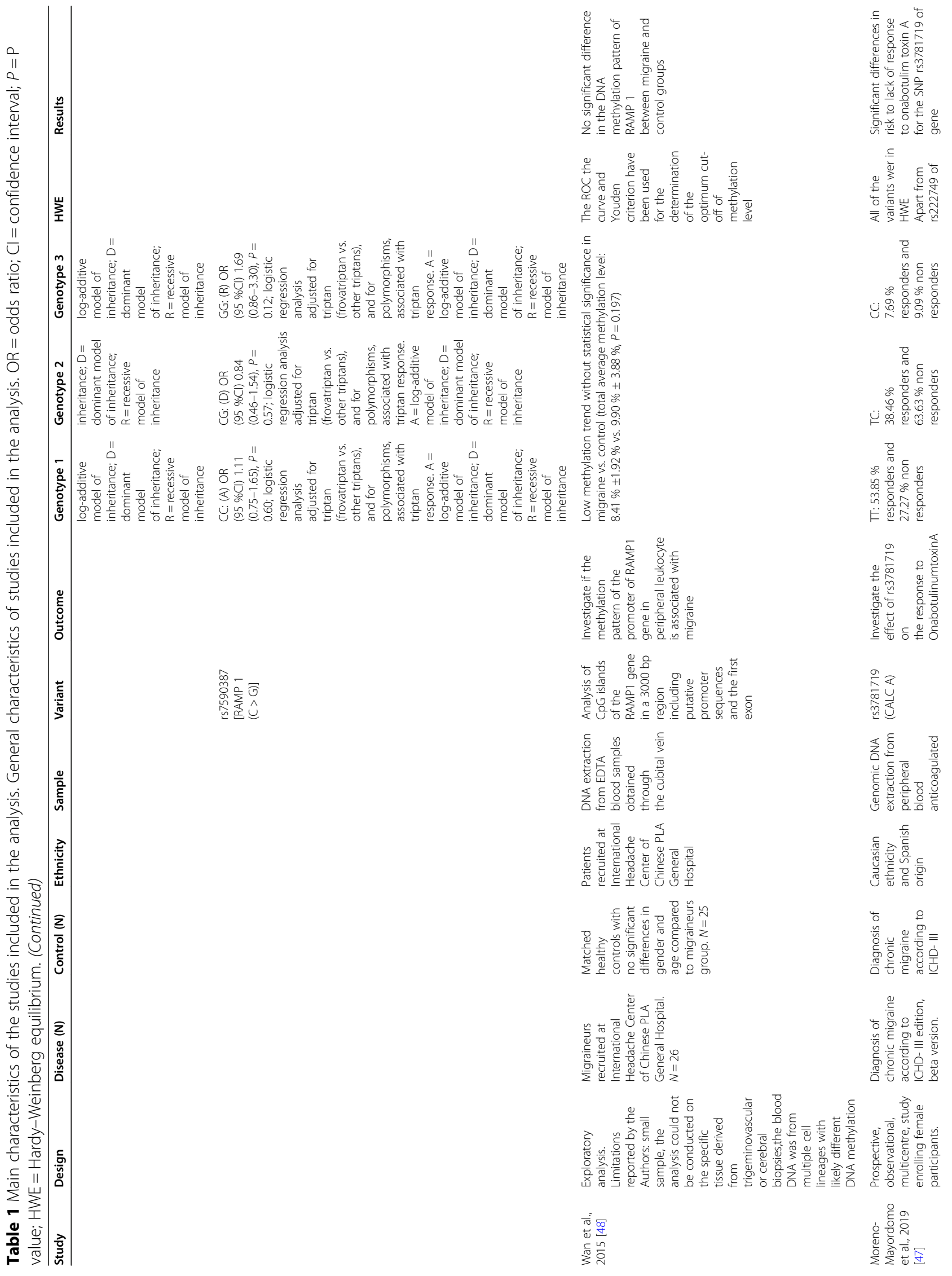




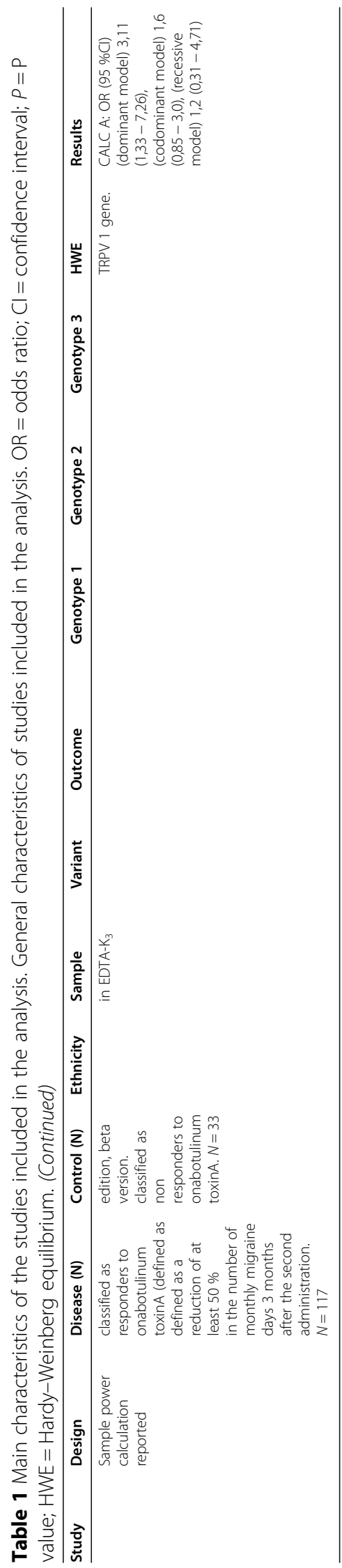


(dominant model) 3,11 (1,33-7,26), (codominant model) 1,6 (0,85-3,0), (recessive model) 1,2 (0,31 $4,71)$ ] in the study by Moreno-Mayordomo and coworkers [47].

\section{Polymorphisms of the gene encoding RAMP 1}

The SNP rs3754701, in the promoter region, and the rs7590387 of the gene encoding RAMP1 have been investigated for the first time by Sutherland and collaborators [45], but no significant associations with migraine susceptibility have been identified. The SNP rs3754701 has been tested also with the SNP rs7590387 of RAMP 1 in migraineurs not presenting aura for association with response to triptans and as risk factors for $\mathrm{MOH}$ by Cargnin et al., [46]. Using the log-additive, the dominant and the recessive model of inheritance response to triptans has not been correlated, but the rs7590387G allele and the rs7590387GG genotype reduce significantly the risk of transformation of episodic migraine into $\mathrm{MOH}$ [OR (95\%CI) (R) $0.27(0.13-0.57) P=0.0002)$ ] [46]. Incidentally, in patients affected by migraine a methylation trend (lower in females) at the promoter region of the gene encoding RAMP 1 without significant differences in the DNA methylation level has been detected by Wan et al., [48].

\section{Assessment of quality of the studies}

The quality of the studies included in the present systematic analysis has been assessed following the HuGE systematic reviews and meta-analyses risk-of-bias score for genetic association studies [38] considering the following 4 outcomes: (1) Information bias, evaluating the accuracy of diagnosis of migraine, the ascertainment of controls matched to cases and the quality of genotyping; (2) Confounding bias, in which all the confounders e.g. population stratification, different ethnicity/gender, sample power calculation and statistical adjustment for confounders have been considered; (3) Selective reporting of outcomes, i.e. mentioning only significant associations with SNPs; (4) HWE assessment. The studies by Lemos et al., 2010, Guldiken et al., 2013, Sutherland et al., 2013, Cargnin et al., 2015 and Moreno-Mayordomo et al., 2019 do not present risk of bias since the criteria for diagnosis of cases vs. controls have been clearly stated and have followed the criteria of the IHS and of the ICHD in effect at the time of the study. In the other studies, clinical neurological assessment, also according to international criteria [43], has been reported, originating unclear risk of bias. The ascertainment of sex, age and ethnicity matched controls has been mentioned in the study by Lemos et al., 2010, Menon et al., 2011, Sutherland et al., 2013, Cargnin et al., 2015, Wan et al., 2015. In the study by Moreno-Mayordomo et al., 2019 both responders and non responders have been determined as chronic migraineurs using the ICHD-III edition, beta version. The demographic and clinical characteristics between case and control groups have resulted comparable, or subjected to further subgroup analyses, in the studies by Cargnin et al., Lemos et al., Menon et al., and Moreno-Mayordomo et al. Apart from multiple comparisons, adjusted analyses for confounding effects, e.g. for triptan type or for SLC6A4 STin2 VNTR and COMT val158me polymorphisms associated to trip$\tan$ response [46], have been conducted in the studies by Cargnin et al., 2015, Moreno-Mayordomo et al., 2019, Menon et al., 2011 and Wan et al., 2015. Weak linkage disequilibrium, indirect genetic association with the true causal variant [49], has been reported in the study by Lemos et al., 2010. Linkage disequilibrium has been performed also in the study by Cargnin et al. and in the study by Sutherland et al. The methods of genotyping have been reported by all the studies. Compromised quality of DNA and successful genotyping have been reported and samples removed from analysis where occurring, e.g. Lemos et al., Menon et al., and Sutherland et al. On the contrary, no discrepancies in genotyping, that had even been re-conducted for validation in about $10 \%$ of the samples, have been found in the study by Cargnin and coworkers. In the study by Guldiken and collaborators smoking and family history of vascular disease were significantly more frequent in the migraine group, but no analysis adjusted for confounders has been reported. In the study by Moreno-Mayordomo and coworkers two of the considered variables have resulted to present significant differences after correction for multiple comparisons between the groups of responders and non-responders. In the study by Cargnin and collaborators, the Authors report that the possibility that some individuals present in the control sample might be affected by $\mathrm{MOH}$ cannot be excluded, but that this fraction would be unlikely to be higher than that observed in the general population. The sample power calculation has been reported in the studies by Lemos et al., Menon et al., Sutherland et al., Cargnin et al., Wan et al., and Moreno-Mayordomo et al. However, in the study by Lemos et al., the Authors highlight that sample size is a limitation of the study not providing enough power to detect a variant with an $\mathrm{OR}<1.5$ and that the study had a power of $64 \%$ to detect an association with the included sample (for a nominal significance level of 0.05). Cargnin et al. report that the study is underpowered to detect small genetic main effects but, its power is sufficient for medium-large effect sizes of clinical relevance. Wan et al. state that sample size calculation could not be accurate since it was the first study investigating RAMP1 methylation pattern and that, therefore, a wider cohort is needed. Other limitations mentioned by Wan et al. are relative to blood DNA sample coming from 
multiple cell lineages with likely different DNA methylation and the issue concerned with the impossibility to conduct the analysis on the specific tissue derived from trigeminovascular or cerebral biopsies. All the studies are devoid of reporting bias. The HWE has been assessed in all the studies and ROC curve and Youden criterion have been used for the determination of the optimum cut-off of methylation level in the study by Wan et al., but the $\mathrm{P}_{\mathrm{HWE}}$ values have been reported only in the studies by Cargnin et al. and Menon et al.

\section{Discussion and conclusions}

Interest in migraine and in SNPs likely linked to susceptibility to its development and clinical features has been growing over the last years. However, little is known about the clinical relevance of these polymorphisms and their effect on the response to anti-migraine treatment. Moreover, the new era of migraine preventative treatments has been opened by the anti-CGRP(R) mAbs, but some $40 \%$ non-responders still represent a pharmacological unmet need. This is the first systematic review that intends to identify SNPs affecting different segments of the CGRP pathway and assessing how they can influence migraine from its development, to the presence of aura and to the efficacy and safety of treatment. From an initial screening of the 800 records identified through database searching, only 7 studies met the inclusion criteria. This is the first obvious red flag that SNPs concerned with the CGRP signaling of clinical relevance are poorly investigated. Within these 7 studies for a total of 2413 patients SNPs and methylation affecting CALC A and RAMP 1 genes have been detected and the most recurring is the rs3781719 exchange $(\mathrm{T}>\mathrm{C})$, a single nucleotide variation (SNV) originating a $2 \mathrm{~KB}$ upstream variant in the CALC A gene promoter, of which clinical significance is not determined [50]. From our analysis no significant association has been found between the SNV rs3781719 and the clinical characteristics of migraine manifestation, i.e. increased frequency of migraine, presence of aura and gender differences [45]. Not even any correlations with responsiveness to triptans under the log-additive, the dominant and the recessive model of inheritance have been highlighted [46], but it has been associated with an increased risk of lack of response to onabotulinumtoxinA [51]. These results have been obtained in population of different gender and ethnicity, being Australian [45], Italian [46] and females of Spanish origin [51]. However, from the study by Guldiken and coworkers we have learnt that the rs3781719 T$692 \mathrm{C}$ is not associated to migraine attacks frequency and severity and to the occurrence of aura in Turkish females [52]. Incidentally, the effects of CALC A rs3781719 $(-692 \mathrm{~T}>\mathrm{C})$ have been studied in Chinese Han women with chronic postsurgical pain 6 months after cesarean section. [53]. The rs3781719C allele has been demonstrated to represent a risk factor for this postoperative chronic pain condition [53]. According to our analysis, the second most investigated SNP affecting the CGRP pathway is the rs7590387 of RAMP 1 locus $[45,46]$, that is another SNV $(C>G)$, also in this case without a demonstrated clinical significance [54]. The latter had been previously investigated in a genome-wide association study finding correlation with migraine [55], but these data had not been published. Although no significant association with migraine susceptibility in general and both with and without aura [45] and with the response to triptans in all the three genotypes under study [46], the rs7590387G allele and the rs7590387GG genotype have been found to reduce significantly the risk of transformation $\mathrm{MOH}$ [46]. Interestingly, the relationship of this SNP with cerebral infarction has been examined in a Japanese population, suggesting the T-A-C haplotype to represent a genetic marker for cerebral infarction [56]. Only one study investigating the methylation pattern with regard to CGRP pathway has resulted from the search. The results of this systematic analysis must be interpreted based on the characteristics of the included studies. In fact, it is important to notice that the genetic variations examined are different within the studies and, also when the same SNP has been investigated, the outcomes considered differed markedly among these studies. Also, the methods of analysis of the results do not make comparison feasible. Moreover, they have been performed on populations not comparable for gender and ethnicity, thus not allowing the generalization of the results. Although the study design is the case-control typical to test for direct genetic associations and the overall quality of the studies meet lot of the HuGe criteria, some concern has been raised: the ascertainment of diagnosis in cases and the matching with controls is not always efficient and clearly reported; the features of migraine considered are different; the adjustement for confounders is not always present and not in each study all the parameters assessing the HWE and efficiency of genotyping are reported completely. Together with the described heterogeneity between the studies, the small sample size and the lack of an adequate number of references for power calculation is a fundamental issue strengthening the need for more investigation in this research field. This is more evident for the exploratory analysis study by Wan et al. Often genetic association studies show initial significant association, but the latter needs to be confirmed by replication studies. These studies must be adequately powered to detect true associations, also in case of small effect conferred by genotype or allele, in order to exclude the risk of falsepositive findings arisen by chance or systematic bias; unfortunately, frequently this does not occur [49]. To 
provide a hypothesis of functional significance of the polymorphisms found in the context of CGRP signalling and migraine, it is mandatory to consider that the data that have resulted close to significance but without reaching it may have been affected by: (1) the small sample size of the cohorts, underpowered to detect small genetic main effects and for further stratification analysis; (2) the confounding influence of environmental factors. However, the results of this systematic review suggest a role of rs7590387GG of RAMP1 in the transformation of episodic migraine into $\mathrm{MOH}$. Interestingly, the collected data have highlighted that rs3781719 of gene CALC A influences the response to onabotulinumtoxinA. Moreover, the first comprehensive genetic association study of patients with chronic and highfrequency migraine has underscored rs2956 of CALC A gene and rs302680 of RAMP1 to be nominally associated with chronic migraine, although these associations have not resulted significant in the replication stage [57]. Therefore, a role of genetic variants affecting the CGRP pathway and, thus the responsiveness to therapeutics, emerges from this systematic review and it warrants further investigation. Remarkably, no genetic association studies investigating the possible effect of CGRP SNPs on the responsiveness to the most recent pharmacological approaches, i.e. anti-CGRP(R) mAbs, gepants and ditans, have been found, apart from the ongoing INTE RROGATE trial. The latter mAbs are a fundamental weapon in the arsenal of migraine therapy even more for difficult-to-treat patients who do not find relief from treatment. In fact, the efficacy of mAbs targeting CGRP in refractory patients has provided very encouraging results [58]. Furthermore, these mAbs are well tolerated and titres of neutralizing and not anti-mAbs antibodies that have been reported were low and not affecting significantly efficacy and safety $[58,59]$. Therefore, the existence of clinically relevant genetic variants along the CGRP signaling deserves further investigation since these could account for some percentage of non responders to treatment with mAbs. In addition, several conditions may lead to lack of responsiveness with consequent persistent migraine [60]: it is possible that CGRP is not completely inhibited in its action or that, although fully blocked, adrenomedullin can still induce vasodilation through heteromerization of its receptor with $\operatorname{CGRP}(\mathrm{R})$. Furthermore, the role of other peptides, e.g. pituitary adenylate cyclase-activating polypeptide (PACAP) or the vasoactive intestinal peptide (VIP) deserves investigation [60]. An attempt to predict the responders to erenumab has highlighted that a lower baseline mean blood flow velocity in cerebral arteries is associated to increased effectiveness, with cerebral blood flow increase after treatment in good responders [61]. Incidentally, early non responders to galcanezumab can turn into responders in the second/third month [62]. Also, recently an association between iron deposits in the periaqueductal gray of patients suffering from chronic migraine and poor response to onabotulinumtoxinA has been observed [63], pointing at the possible involvement of metal deposits in the responsiveness to anti-migraine treatments. Thus, it is fundamental to understand how SNPs and epigenetic modifications may affect the response to anti-migraine treatment, since most drugs directly or indirectly target the latter pathway, in order to predict treatment efficacy and safety. A window is opening on the association between disabling primary headache and preclinical familial Alzheimer's disease [64]. Moreover, both dementia and migraine are main symptoms of CADASIL (cerebral autosomal dominant arteriopathy with subcortical infarcts and leukoencephalopathy, OMIM\#125,310) [65]. Therefore, the genetic association of these widespread disabling diseases needs to be studied. Furthermore, aged populations need to be included in migraine genetic association studies, as well as in clinical trials for migraine treatment [66-71]. Hence, adequately powered studies following the criteria for rigorous genetic association are needed to provide high quality evidence on the impact of genetic variants affecting CGRP signaling on migraine susceptibility and clinical manifestation and to predict the response to therapy in terms of efficacy and safety.

\section{Abbreviations \\ ACE: angiotensin converting enzyme; AV: arterial vessels; BIOMIGA: BIOmarkers of MIGraine; CALCRL: human G protein-coupled recep- tor calcitonin receptor-like receptor; CDEM: chronic disorders with episodic manifestations; $\mathrm{Cl}$ : confidence interval; CGRP: calcitonin-gene related peptide; COMT: catechol-O-methyltransferase; CSD: cortical spreading depression; Glu: glutamate; HLA: human leukocyte antigens; HuGE: Human Genome Epidemiology; HWE: Hardy-Weinberg equilibrium; mAbs: monoclonal antibodies; MAO: monoamine oxidase; MTHFR: methylenetetrahydrofolate reductase; MTR: methionine synthase; MTRR: methionine synthase reductase; NO: nitric oxide; OR: odds ratio; PICOS: participants/population, interventions, comparisons, outcomes, and study design; PRISMA: Preferred Reporting Items for Systematic reviews and Meta-Analyses; PROSPERO: International prospective register of systematic reviews; RAMP-1: receptor activity- modifying protein; SNP: single-nucleotide polymorphism; SNPS: single- nucleotide polymorphisms; YLD: Years Lived with Disability}

\section{Acknowledgements}

D.S. is a post-doc funded by S. Anna Institute, Crotone, Italy.

\section{Authors' contributions}

All authors have contributed equally to the work. All authors have read and approved the final manuscript.

\section{Funding}

Not applicable. Open Access funding enabled and organized by Projekt DEAL.

Availability of data and materials

All data generated or analysed during this study are included in this published article/Data sharing is not applicable to this article as no datasets were generated or analysed during the current study. 


\section{Declarations}

\section{Ethics approval and consent to participate}

Not applicable.

\section{Consent for publication}

Not applicable.

\section{Competing interest}

The authors declare that they have no competing interests.

\section{Author details}

${ }^{1}$ Pharmacotechnology Documentation and Transfer Unit, Preclinical and Translational Pharmacology, Department of Pharmacy, Health and Nutritional Sciences, University of Calabria, 87036 Rende, Italy. ${ }^{2}$ Regional Center for Serious Brain Injuries, S. Anna Institute, Crotone, Italy. ${ }^{3}$ Department of Health Sciences, University "Magna Graecia" of Catanzaro, 88100 Catanzaro, Italy. ${ }^{4}$ German Center for Neurodegenerative Diseases (DZNE), Bonn, Germany.

\section{Received: 23 May 2021 Accepted: 12 July 2021}

Published online: 30 July 2021

\section{References}

1. Headache Classification Committee of the International Headache Society (IHS) The International Classification of Headache Disorders, 3rd edition. Cephalalgia. 2018;38(1):1-211

2. Global regional, national burden of neurological disorders, 1990-2016: a systematic analysis for the Global Burden of Disease Study 2016. Lancet Neurol. 2019:18(5):459-480

3. Leonardi M, Grazzi L, D'Amico D, Martelletti P, Guastafierro E, Toppo C et al (2021) Global Burden of Headache Disorders in Children and Adolescents 2007-2017. International Journal of Environmental Research Public Health 18(1):250

4. Finding promising cures (2021) for migraine: targeting neuropeptides. EBioMedicine 63:103225

5. Haut SR, Bigal ME, Lipton RB (2006) Chronic disorders with episodic manifestations: focus on epilepsy and migraine. Lancet Neurol 5(2):148-157

6. Bigal ME, Liberman JN, Lipton RB (2006) Age-dependent prevalence and clinical features of migraine. Neurology 67(2):246-251

7. Sessle BJ, Hu JW, Amano N, Zhong G (1986) Convergence of cutaneous, tooth pulp, visceral, neck and muscle afferents onto nociceptive and nonnociceptive neurones in trigeminal subnucleus caudalis (medullary dorsal horn) and its implications for referred pain. Pain 27(2):219-235

8. Maleki N, Szabo E, Becerra L, Moulton E, Scrivani SJ, Burstein R et al (2021) Ictal and interictal brain activation in episodic migraine: Neural basis for extent of allodynia. PloS one 16(1):e0244320

9. Burstein R, Cutrer MF, Yarnitsky D (2000) The development of cutaneous allodynia during a migraine attack clinical evidence for the sequential recruitment of spinal and supraspinal nociceptive neurons in migraine. Brain 123(Pt 8):1703-1709

10. Ashina M, Hansen JM, Do TP, Melo-Carrillo A, Burstein R, Moskowitz MA (2019) Migraine and the trigeminovascular system-40 years and counting. Lancet Neurol 18(8):795-804

11. Messlinger K, Lennerz JK, Eberhardt M, Fischer MJ (2012) CGRP and NO in the trigeminal system: mechanisms and role in headache generation. Headache 52(9):1411-1427

12. De Matteis E, Guglielmetti M, Ornello R, Spuntarelli V, Martelletti P, Sacco S (2020) Targeting CGRP for migraine treatment: mechanisms, antibodies, small molecules, perspectives. Expert Rev Neurother 20(6):627-641

13. Goadsby PJ, Holland PR, Martins-Oliveira M, Hoffmann J, Schankin C, Akerman S (2017) Pathophysiology of Migraine: A Disorder of Sensory Processing. Physiological reviews 97(2):553-622

14. Scuteri D, Adornetto A, Rombolà L, Naturale MD, Morrone LA, Bagetta G et al (2019) New Trends in Migraine Pharmacology: Targeting Calcitonin Gene-Related Peptide (CGRP) With Monoclonal Antibodies. Front Pharmacol $10: 363$

15. Scuteri D, Corasaniti MT, Tonin P, Bagetta G (2019) Eptinezumab for the treatment of migraine. Drugs of today 55(11):695-703

16. Ashina M (2019) The most important advances in headache research in 2018. Lancet Neurol 18(1):5-6
17. Giacovazzo M, Valeri M, Piazza A, Torlone N, Bernoni RM, Martelletti P et a (1987) Elevated frequency of HLA shared-haplotypes in migraine families. Headache 27(10):575-577

18. Martelletti P, Lulli P, Morellini M, Mariani B, Pennesi G, Cappellacci S et al (1999) Chromosome 6p-encoded HLA-DR2 determination discriminates migraine without aura from migraine with aura. Hum Immunol 60(1):69-74

19. Roecklein KA, Scher Al, Smith A, Harris T, Eiriksdottir G, Garcia M et al (2013) Haplotype analysis of the folate-related genes MTHFR, MTRR, and MTR and migraine with aura. Cephalalgia 33(7):469-482

20. Pomes LM, Guglielmetti M, Bertamino E, Simmaco M, Borro M, Martelletti P (2019) Optimising migraine treatment: from drug-drug interactions to personalized medicine. J Headache Pain 20(1):56

21. Piane M, Lulli P, Farinelli I, Simeoni S, De Filippis S, Patacchioli FR et al (2007) Genetics of migraine and pharmacogenomics: some considerations. J Headache Pain 8(6):334-339

22. Simmaco M, Borro M, Missori S, Martelletti P (2009) Pharmacogenomics in migraine: catching biomarkers for a predictable disease control [corrected]. Expert Rev Neurother 9(9):1267-1269

23. Capi M, Gentile G, Lionetto L, Salerno G, Cipolla F, Curto M et al (2018) Pharmacogenetic considerations for migraine therapies. Expert Opin Drug Metab Toxicol 14(11):1161-1167

24. Schürks M, Kurth T, Stude P, Rimmbach C, de Jesus J, Jonjic M et al (2007) G protein beta3 polymorphism and triptan response in cluster headache. Clin Pharmacol Ther 82(4):396-401

25. Gentile G, Borro M, Lala N, Missori S, Simmaco M, Martelletti P (2010) Genetic polymorphisms related to efficacy and overuse of triptans in chronic migraine. J Headache Pain 11(5):431-435

26. Cargnin S, Magnani F, Viana M, Tassorelli C, Mittino D, Cantello R et al (2013) An opposite-direction modulation of the COMT Val158Met polymorphism on the clinical response to intrathecal morphine and triptans. The journal of pain 14(10):1097-1106

27. Taylor FR (2018) Antigens and Antibodies in Disease With Specifics About CGRP Immunology. Headache 58(Suppl 3):230-237

28. Takai T (2002) Roles of Fc receptors in autoimmunity. Nature reviews Immunology 2(8):580-592

29. Nimmeriahn F, Gordan S, Lux A (2015) FcgammaR dependent mechanisms of cytotoxic, agonistic, and neutralizing antibody activities. Trends Immunol 36(6):325-336

30. Kaifu T, Nakamura A (2017) Polymorphisms of immunoglobulin receptors and the effects on clinical outcome in cancer immunotherapy and other immune diseases: a general review. Int Immunol 29(7):319-325

31. Dolcetti $R$ (2017) Predictive Value of FcR Polymorphisms: A Further Step on the Long and Winding Road to Application. JAMA oncology 3(3):342-343

32. Eising E, van den Maagdenberg NAD, Ferrari AM (2013) MD. Epigenetic mechanisms in migraine: a promising avenue? BMC Med 11:26

33. Liberati A, Altman DG, Tetzlaff J, Mulrow C, Gøtzsche PC, loannidis JP et al (2009) The PRISMA statement for reporting systematic reviews and metaanalyses of studies that evaluate health care interventions: explanation and elaboration. PLoS Med 6(7):e1000100

34. Moher D, Liberati A, Tetzlaff J, Altman DG, Group P (2009) Preferred reporting items for systematic reviews and meta-analyses: the PRISMA statement. PLoS Med 6(7):e1000097

35. Little JHJ e. The HuGENet ${ }^{\mathrm{TM} H u G E}$ review handbook, Version 1.0. Ottawa: University of Ottawa; 2006

36. Jones J, Hunter D (1995) Qualitative Research: Consensus methods for medical and health services research. BMJ 311(7001):376-380

37. Ryan R, Group. CCaCR. Cochrane Consumers and Communication Review Group: data synthesis and analysis. http://cccrg.cochrane.org, 13 March 2019 (accessed DATE)

38. Thakkinstian A, McKay GJ, McEvoy M, Chakravarthy U, Chakrabarti S, Silvestri $\mathrm{G}$ et al (2011) Systematic review and meta-analysis of the association between complement component 3 and age-related macular degeneration: a HuGE review and meta-analysis. Am J Epidemiol 173(12):1365-1379

39. Loannidis JP, Boffetta P, Little J, O'Brien TR, Uitterlinden AG, Vineis $P$ et al (2008) Assessment of cumulative evidence on genetic associations: interim guidelines. Int J Epidemiol 37(1):120-132

40. An X, Yu Z, Fang J, Lin Q, Lu C, Ma QL et al (2017) Association of CALCA and RAMP1 gene polymorphisms with migraine in a chinese population. Neurology Asia 22(3):221-225

41. Louter MA, Fernandez-Morales J, De Vries B, Winsvold B, Anttila V, Fernandez-Cadenas I et al (2015) Candidate-gene association study 
searching for genetic factors involved in migraine chronification. Cephalalgia 35(6):500-507

42. Lemos C, Mendonca D, Pereira-Monteiro J, Barros J, Sequeiros J, Alonso I et al (2010) BDNF and CGRP interaction: Implications in migraine susceptibility. Cephalalgia 30(11):1375-1382

43. Menon S, Buteri J, Roy B, Murrell M, Quinlan S, Macmillan JC et al (2011) Association study of calcitonin gene-related polypeptide-alpha (CALCA) gene polymorphism with migraine. Brain research 1378:119-124

44. Guldiken B, Sipahi T, Tekinarslan R, Kabayel L, Ozkan H, Unlu A et al (2013) Calcitonin gene related peptide gene polymorphism in migraine patients. Can J Neurol Sci 40(5):722-725

45. Sutherland HG, Buteri J, Menon S, Haupt LM, Macgregor EA, Lea RA et al (2013) Association study of the calcitonin gene-related polypeptide-alpha (CALCA) and the receptor activity modifying 1 (RAMP1) genes with migraine. Gene 515(1):187-192

46. Cargnin S, Pautasso C, Viana M, Sances G, Mittino D, Cantello R et al (2015) Association of RAMP1rs7590387 with the risk of migraine transformation into medication overuse headache. Headache 55(5):658-668

47. Moreno-Mayordomo R, Ruiz M, Pascual J, de la Sacristana MG, Vidriales I, Sobrado M et al. CALCA and TRPV1 genes polymorphisms are related to a good outcome in female chronic migraine patients treated with OnabotulinumtoxinA. J Headache Pain. 2019;20:39. https://doi.org/10.1186/ s10194-019-0989-9

48. Wan DJ, Hou L, Zhang XF, Han X, Chen M, Tang WJ et al. DNA methylation of RAMP1 gene in migraine: an exploratory analysis. J Headache Pain. 2015: 16:90. https://doi.org/10.1186/s10194-015-0576-7

49. Lewis CM, Knight J (2012) Introduction to genetic association studies. Cold Spring Harbor Protoc 2012(3):297-306

50. (NCBI) dSGV. https://www.ncbi.nlm.nih.gov/snp/rs3781719\#variant_details

51. Moreno-Mayordomo R, Ruiz M, Pascual J, Gallego de la Sacristana M, Vidriales I, Sobrado M et al (2019) CALCA and TRPV1 genes polymorphisms are related to a good outcome in female chronic migraine patients treated with OnabotulinumtoxinA. J Headache Pain 20(1):39

52. Guldiken B, Sipahi T, Tekinarslan R, Kabayel L, Ozkan H, Unlu A et al (2013) Calcitonin gene related peptide gene polymorphism in migraine patients. The Canadian journal of neurological sciences Le journal canadien des sciences neurologiques 40(5):722-725

53. Ma G, Yang J, Zhao B, Huang C, Wang R (2019) Correlation between CCL2 CALCA, and CX3CL1 gene polymorphisms and chronic pain after cesarean section in Chinese Han women: A case control study. Medicine 98(34):e16706

54. (NCBI) dSGV. https://www.ncbi.nlm.nih.gov/snp/rs7590387\#variant_details

55. Cox HC, Lea RA, Bellis C, Carless M, Dyer TD, Curran J et al (2012) A genome-wide analysis of 'Bounty' descendants implicates several novel variants in migraine susceptibility. Neurogenetics 13(3):261-266

56. Nakazato T, Nakayama T, Naganuma T, Sato N, Fu Z, Wang Z et al (2010) Haplotype-based case-control study of receptor (calcitonin) activity-modifying protein-1 gene in cerebral infarction. J Hum Hypertens 24(5):351-358

57. Louter MA, Fernandez-Morales J, de Vries B, Winsvold B, Anttila V, Fernandez-Cadenas I et al (2015) Candidate-gene association study searching for genetic factors involved in migraine chronification. Cephalalgia 35(6):500-507

58. Charles A, Pozo-Rosich P (2019) Targeting calcitonin gene-related peptide: a new era in migraine therapy. Lancet 394(10210):1765-1774

59. Cohen JM, Ning X, Kessler Y, Rasamoelisolo M, Campos VR, Seminerio MJ et al (2021) Immunogenicity of biologic therapies for migraine: a review of current evidence. J Headache Pain 22(1):3

60. de Vries T, MaassenVanDenBrink A (2019) CGRP-targeted antibodies in difficult-to-treat migraine. Nature Reviews Neurology 15(12):688-689

61. Nowaczewska M, Straburzyński M, Meder G, Kaźmierczak W. Changes in Cerebral Blood Flow after Erenumab Treatment in Good and NonResponders-A Pilot Study of Migraine Patients. Journal of clinical medicine. 2021;10(11). https://doi.org/10.3390/jcm10112523

62. Goadsby PJ, Dodick DW, Martinez JM, Ferguson MB, Oakes TM, Zhang Q et al (2019) Onset of efficacy and duration of response of galcanezumab for the prevention of episodic migraine: a post-hoc analysis. J Neurol Neurosurg Psychiatry 90(8):939-944

63. Domínguez Vivero C, Leira Y, Saavedra Piñeiro M, Rodríguez-Osorio $X$, Ramos-Cabrer P, Villalba Martín C et al. Iron Deposits in Periaqueductal Gray Matter Are Associated with Poor Response to OnabotulinumtoxinA in Chronic Migraine. Toxins. 2020;12(8):479. https://doi.org/10.3390/toxins12 080479
64. Ringman JM, Romano JD, Medina LD, Rodriguez-Agudelo Y, Schaffer B, Varpetian A et al (2008) Increased prevalence of significant recurrent headache in preclinical familial Alzheimer's disease mutation carriers. Dement Geriatr Cogn Disord 25(4):380-384

65. Muiño E, Maisterra O, Jiménez-Balado J, Cullell N, Carrera C, Torres-Aguila NP et al (2021) Genome-wide transcriptome study in skin biopsies reveals an association of E2F4 with cadasil and cognitive impairment. Scientific reports 11(1):6846

66. Scuteri D, Adornetto A, Rombolà L, Naturale MD, De Francesco AE, Esposito $S$ et al (2020) Pattern of triptans use: a retrospective prescription study in Calabria, Italy. Neural regeneration research 15(7):1340-1343

67. Scuteri D, Piro B, Morrone LA, Corasaniti MT, Vulnera M, Bagetta G (2017) The need for better access to pain treatment: learning from drug consumption trends in the USA. Functional neurology 22(4):229-230

68. Scuteri D, Garreffa MR, Esposito S, Bagetta G, Naturale MD, Corasaniti MT (2018) Evidence for accuracy of pain assessment and painkillers utilization in neuropsychiatric symptoms of dementia in Calabria region, Italy. Neural regeneration research 13(9):1619-1621

69. Scuteri D, Vulnera M, Piro B, Bossio RB, Morrone LA, Sandrini G et al (2021) Pattern of treatment of behavioural and psychological symptoms of dementia and pain: evidence on pharmacoutilization from a large realworld sample and from a centre for cognitive disturbances and dementia. Eur J Clin Pharmacol 77(2):241-249

70. Scuteri D, Rombolà L, Morrone LA, Bagetta G, Sakurada S, Sakurada T et al. Neuropharmacology of the Neuropsychiatric Symptoms of Dementia and Role of Pain: Essential Oil of Bergamot as a Novel Therapeutic Approach. International journal of molecular sciences. 2019; 20(13):3327. https://doi. org/10.3390/ijms20133327

71. Rombolà L, Scuteri D, Adornetto A, Straface M, Sakurada T, Sakurada S et al (2019) Anxiolytic-Like Effects of Bergamot Essential Oil Are Insensitive to Flumazenil in Rats. Evidence-based complementary alternative medicine: eCAM 2019:2156873

\section{Publisher's Note}

Springer Nature remains neutral with regard to jurisdictional claims in published maps and institutional affiliations.

Ready to submit your research? Choose BMC and benefit from

- fast, convenient online submission

- thorough peer review by experienced researchers in your field

- rapid publication on acceptance

- support for research data, including large and complex data types

- gold Open Access which fosters wider collaboration and increased citations

- maximum visibility for your research: over $100 \mathrm{M}$ website views per year

At BMC, research is always in progress.

Learn more biomedcentral.com/submissions 